\title{
Safety challenges related to the use of sedation and general anesthesia in pediatric patients undergoing magnetic resonance imaging examinations
}

\author{
Maddy Artunduaga ${ }^{1,2}$ (D) C. Amber Liu ${ }^{3,4} \cdot$ Cara E. Morin ${ }^{5}$ S Suraj D. Serai ${ }^{6,7}$ - Unni Udayasankar ${ }^{8}$. \\ Mary-Louise C. Greer ${ }^{9,10} \cdot$ Michael S. Gee ${ }^{4,11}$
}

Received: 10 September 2020 / Revised: 17 January 2021 / Accepted: 1 March 2021 / Published online: 16 April 2021

(C) The Author(s), under exclusive licence to Springer-Verlag GmbH Germany, part of Springer Nature 2021

\begin{abstract}
The use of sedation and general anesthesia has facilitated the significant growth of MRI use among children over the last years. While sedation and general anesthesia are considered to be relatively safe, their use poses potential risks in the short term and in the long term. This manuscript reviews the reasons why MRI examinations require sedation and general anesthesia more commonly in the pediatric population, summarizes the safety profile of sedation and general anesthesia, and discusses an amalgam of strategies that can be implemented and can ultimately lead to the optimization of sedation and general anesthesia care within pediatric radiology departments.
\end{abstract}

Keywords Child life $\cdot$ Children $\cdot$ Dog therapy $\cdot$ General anesthesia $\cdot$ Magnetic resonance imaging $\cdot$ Safety $\cdot$ Sedation $\cdot$ Technique

\section{Introduction}

Magnetic resonance imaging (MRI) is increasingly used in a variety of pediatric conditions because of its high soft-tissue contrast resolution and utility for acquiring multiplanar images to evaluate the anatomy and function of organs in the body without the use of ionizing radiation [1-3]. Although MRI offers several advantages over other imaging modalities, it is limited by long acquisition times and its environment, which involves an enclosed space and inherently loud noises $[1,4]$.

Maddy Artunduaga

maddy.artunduaga@utsouthwestern.edu

1 Pediatric Radiology Division, Department of Radiology,

University of Texas Southwestern Medical Center,

Children's Health Medical Center,

5323 Harry Hines Blvd., CMC F1.02, Dallas, TX 75390, USA

2 Children's Health Medical Center, Dallas, TX, USA

3 Department of Anesthesia, Critical Care and Pain Medicine, Massachusetts General Hospital, Boston, MA, USA

4 Harvard Medical School, Boston, MA, USA

5 Department of Diagnostic Imaging, St. Jude Children's Research Hospital, Memphis, TN, USA
The use of sedation and general anesthesia has facilitated the assessment of pediatric patients with MRI, particularly in children older than 3-6 months and younger than 6 years of age. Sedation and general anesthesia are typically used in infants and young children with lengthy MRI examinations because remaining motionless and following breathing commands, which are necessary to obtain the best possible MR image quality, can be challenging during these ages $[1,4]$. Quality control of sedated/anesthetized cases is essential and should be actively performed by radiologists, along with constant communication with MRI technologists, to ensure that

6 Department of Radiology, Children's Hospital of Philadelphia, Philadelphia, PA, USA

7 Perelman School of Medicine at the University of Pennsylvania, Philadelphia, PA, USA

8 Department of Medical Imaging, The University of Arizona Health Sciences, Tucson, AZ, USA

9 Department of Diagnostic Imaging, The Hospital for Sick Children, Toronto, ON, Canada

10 Medical Imaging, University of Toronto, Toronto, ON, Canada

11 Department of Radiology, Massachusetts General Hospital, Boston, MA, USA 
each study is diagnostic prior to its completion and to avoid potential recalls that might require a repeat MRI examination under sedation/general anesthesia.

Although the use of sedation and general anesthesia is considered to be relatively safe, with low reported rates of serious adverse events in institutions with well-organized sedation services, the risk of mild and moderate adverse events at the time of anesthesia remains substantial [5]. Additionally, the potential for long-term effects of anesthetic exposure on subsequent neurodevelopmental outcomes based on preclinical models and some retrospective clinical data has been also raised in recent years [6-9]. These concerns prompted the United States Food and Drug Administration (FDA) to issue a warning in December 2016 to caution the use of repeated and lengthy (i.e. more than $3 \mathrm{~h}$ ) general anesthesia and sedation in children younger than 3 years [10]. Although neurocognition in the developing brain is challenging to assess given its complexity and the multiple contributing factors involved [11], a recent multi-national multi-institutional randomized controlled trial with follow-up at 2 years and 5 years has shown that the use of single and short exposures (i.e. less than $1 \mathrm{~h}$ ) of sedation/general anesthesia in this young pediatric cohort is considered to be safe [6-8]. In light of this, it is also important to highlight that not performing an MRI examination or obtaining suboptimal MR image quality in order to avoid sedation/general anesthesia could be detrimental to patient care, and that the ultimate decision of its use should be based on a risk-benefit assessment.

Over the years, radiology departments around the world have developed innovative techniques to decrease the use of sedation and general anesthesia in pediatric MRI, including feed-and-swaddle, fast-acquisition techniques, certified child life specialists' assessments, mock MRI use and dog-therapy programs, among other initiatives [12-15]. This article offers a comprehensive review of the nuances and safety challenges related to the use of sedation and general anesthesia in the pediatric population, as well as a summary of strategies to overcome these challenges based on the collective experience of the authors' institutions, which have been successful in decreasing overall MRI sedation and general anesthesia utilization rates.

\section{Magnetic resonance imaging system properties impacting tolerance by children}

Several intrinsic MR imaging characteristics affect the tolerance of these examinations by children, and thus image quality. MRI scan duration, which determines the length of time children need to lie still, along with the need to suspend respiration during certain breath-hold acquisitions in body MRI, are significant causes of patient anxiety $[12,16]$. The enclosed MRI environment with a built-in small-bore tunnel [17] where patients lie can lead to claustrophobia [2]. The unfamiliar MRI environment, along with the presence of health care professionals who are unfamiliar to the child, can create fear, agitation and anxiety in young children $[2,4,12]$. In addition, the need for intravenous cannulation for exams requiring gadolinium-based contrast agent can be a cause of anxiety and discomfort in the pediatric population $[2,16]$.

\section{Sedation and general anesthesia use in the pediatric population}

Pediatric sedation and anesthesia guidelines have evolved since the first monitoring guidelines were published by the American Academy of Pediatrics Section on Anesthesiology and Pain Medicine in 1985 [18]. Overall, sedation and general anesthesia are a continuum of central nervous system depression states that are induced by medications and allow people to tolerate medical procedures [18]. Depending upon the level of central nervous system depression, the different types of sedation include minimal (awake and calm with cognitive impairment but respondent to verbal stimulation, with regular respirations), moderate (sleepy but still respondent to verbal stimulation or to light tactile stimulation, with slow and shallow respirations) and deep (asleep but respondent to repeated verbal or pain stimulation and possibly requiring ventilatory assistance but with cardiovascular function typically preserved) [18]. With general anesthesia, the loss of consciousness can be accompanied by loss of protective airway reflexes and cardiovascular function [18]. Failure to achieve adequate sedation during procedures, especially in pediatric patients, might result in the need for general anesthesia [18]. In general, vital signs monitoring is necessary because of the potential progression in the depression state from moderate sedation to general anesthesia [18].

The utilization rates of sedation and general anesthesia with MRI are typically higher because other imaging modalities (radiography, US and CT) have much shorter acquisition times [15]. While sedation and general anesthesia have been instrumental to increase the use and clinical importance of MRI in the pediatric population [19], their use also carries important disadvantages including increased risks of adverse events and overdosage, increased length of hospital stay and financial costs, as well as potentially longer wait times for MR scheduling [12, 13, 19-21].

\section{Cognitive outcomes in the developing brain with sedation and general anesthesia use}

Several publications with animal models and some reports with human subjects prompted the FDA to issue a warning in December 2016 to inform the public about the potential 
risks of impaired pediatric neurodevelopment related to repeated or lengthy exposure of general anesthetic and sedation medications during surgeries of procedures in infants and young children up to the age of 3 [10]. Pre-clinical models studies have reported neurotoxicity from neuronal cell death, disrupted neurogenesis, glial death, axon formation anomalies, abnormal behaviors and impaired learning [8]. While propofol, ketamine and volatile anesthetics (e.g., isoflurane) have been specifically used in several of these animal models [22-24], these reports have not been translated clinically [25-27]. To date, studies have failed to provide unequivocal evidence for the role of general anesthesia/sedation in producing neurotoxicity in humans $[8,28-30]$. For instance, although a recent observational clinical study found that repeated exposure to anesthesia/surgery during the first 2 years of age was a significant independent risk factor for the development of learning disabilities and the need for individualized education programs, it concluded that prospective studies are needed to further address this potential association [9]. In addition, there is overall scarce literature that focuses on the effects of general anesthesia exposure in children ages 3 months to 4 years [11].

The most robust collaborative effort so far comes from a randomized controlled trial well-known as the general anesthesia and awake-regional anesthesia (GAS) study. The study, with up to 5 years of follow-up, found strong evidence indicating that short exposures (i.e. less than $1 \mathrm{~h}$ ) to sedation/general anesthesia are not associated with significant neurocognitive or behavioral deficits [8]. This investigation also found no evidence of increased risk between the use of general anesthesia and the diagnosis of attention-deficit hyperactivity disorder (ADHD) or other learning disabilities (e.g., autism spectrum disorder), although this association was limited given the short patient follow-up because these diagnoses are typically made in older children [8]. However, the role of inhalational anesthetic exposure on neurocognitive outcomes in children requiring multiple anesthetics for MRIs continues to be unclear because the GAS study excluded children with preexisting congenital conditions, such as cardiovascular or neurologic disease, which encompass many pediatric patients who already have an inherent risk for poorer neurodevelopmental outcomes and typically undergo multiple MRI examinations.

Investigations to date have several limitations because of their predominant retrospective design with small cohorts and inherent reduced statistical power, the presence of several confounding factors (e.g., underlying conditions such as congenital heart disease and minor versus major surgeries), selection bias, as well as challenges and variability while assessing outcomes and patient loss at follow-up [9, 11]. Future investigations with more rigorous study designs, outcomes evaluation, incorporation of neuroimaging and biomarkers assessment need to be pursued to support a more definitive association between neurotoxicity and general anesthesia/sedation use in humans $[8,11]$.

\section{Non-neurocognitive risks related to the use of sedation and general anesthesia}

Serious adverse events related to pediatric sedation/ general anesthesia outside the operating room are rare $[31,32]$. The Pediatric Sedation Research Consortium (PSRC) has a large database of consecutive pediatric sedation/anesthesia encounters at $>30$ centers [33]. Among nearly 50,000 sedation/anesthesia events, no deaths occurred and only two cardiac arrests were reported, with both patients recovering quickly [31]. Airwayrelated events (stridor, laryngospasm, airway obstruction, wheezing or central apnea) occurred in approximately 1 in 65 children undergoing sedation/anesthesia. One in 70 propofol administrations required airway and ventilation interventions of various severity [31]. A subsequent analysis from the PSRC of nearly 140,000 sedation encounters showed 0 deaths, 10 aspirations and 75 major complications [32]. This analysis specifically delved into the relationship of nil per os (NPO, or pre-study fasting) status and aspiration, finding that NPO status was not an independent risk predictor of aspiration or other major complications in this large dataset [32].

Young infants, especially those who were born pre-term, are at higher risk of postoperative apnea related to sedation/ anesthesia, long thought to be worse in the setting of general anesthesia [34]. A multi-institutional prospective study of 722 infants (younger than 60 weeks of postmenstrual age) randomized infants to receive regional anesthesia or general anesthesia [6]. Overall, the rate of apnea events was very low, occurring in $3 \%$ of participants with no evidence that regional anesthesia reduced the overall risk of apnea. However, regional anesthesia was associated with reduced risk of early postoperative apnea, the degree of postoperative desaturations, and the level of intervention for apnea [6]. Increasingly, propofol is a common agent for pediatric sedation/anesthesia in radiology procedures [32, 35-37]. A retrospective review of 304 infants younger than 6 months undergoing propofol administration for imaging studies showed that 4.3\% (13/ $304)$ had severe adverse events, with $18.4 \%$ (56/304) requiring interventions for airway-related events [36]. The PSRC has also shown that infants younger than 6 months undergoing propofol sedation/anesthesia are at increased risk of adverse pulmonary events, with an odds ratio of 1.77 compared to the reference group of 8- to 18 -year-olds $(P<0.001)$ [31]. In addition to airway-related complications, the use of sedation and general anesthesia poses a significant risk for the development of hypoglycemia in neonates and infants $[7,8]$. 


\section{General anesthesia care nuances in pediatric patients}

General anesthesia outside the operating room is a rapidly developing field, and many efforts have been made in the anesthesia community to improve its safety. Pediatric anesthesia is a specialized field within anesthesia because of the many anatomical, airway and physiological differences between infants/children and adults. Anesthesia for the pediatric MRI is especially challenging because of several factors, discussed next.

\section{Anesthesia techniques for pediatric magnetic resonance imaging}

Because of MRI's high noise level and spatial constraints, pediatric patients who are undergoing MRI and require anesthesia tend to require general anesthesia rather than light sedation to minimize scan stopping and failure rate. The advantage of general anesthesia for MRI is that it is independent of a child's ability to cooperate. However, because of the limited access to the pediatric patient in the MRI environment, and the limited time it takes for hypoxia to occur in such children because of their increased metabolic demand, the anesthesiologist caring for the pediatric patient on the MRI scanner should be a specialist in pediatric airway and anesthetic management.

Several general anesthetic techniques can be used during MRI. Inhalational anesthetics such as sevoflurane, intravenous anesthetics such as propofol, or a combination of the two, are the most commonly used. Inhalational anesthetics should be administered utilizing an MRI safe/conditional anesthesia machine and vaporizer. If intravenous anesthesia is used, it should be administered using MRI safe/conditional infusion pumps and specialized tubing. Airway management techniques can include natural airway with spontaneous breathing and supplemental oxygen by nasal cannula, as well as the more invasive laryngeal mask airway and endotracheal tube, depending on the child's comorbidities, anatomy and fasting status. Complex airway management including fiberoptic intubation or video laryngoscopy should be performed in a controlled environment outside zone IV because these advanced airway equipment devices are usually not MRI-compatible. In general, the most commonly used airway techniques for MRI studies are natural airway and laryngeal mask airway. Ultimately, the choice of airway management depends upon the child's age, size and anatomy as well as the anesthesiologist's preference and the availability of personnel who could help the anesthesiologist if an airway emergency situation develops.

\section{Routine anesthesia equipment}

All children undergoing MRI under anesthesia should be monitored in a manner consistent with the American Society of Anesthesiologists recommended monitors. For pediatric patients under general anesthesia, these include electrocardiogram, blood pressure, pulse oximetry and continuous end-tidal carbon dioxide. The pediatric anesthesiologist should be familiar with the expected limitations of available monitoring equipment in the MRI setting. All monitors used in zone IV must be safe/conditional for MRI. A monitor should be available for the pediatric anesthesiologist to view vital signs outside of zone IV when the scan is occurring. Care should be taken when positioning the electrocardiogram and pulse oximetry to avoid burns.

On the anesthesia workstation, any equipment with metallic materials must be removed because they can impair image quality and cause undesirable safety events. Any anesthesia workstation that remains in an MRI environment during scanning should fulfill MRI criteria to meet the highly specialized respective needs. Specifically, anesthesia machines, gas vaporizers, capnography, pulse oximetry, electrocardiogram monitor and blood pressure measurement must all be MRIcompatible because they will remain with the child during scanning. Other routine anesthesia equipment such as airway devices, laryngoscopes, anesthesia circuits, intravenous tubing, syringes and medications packages/vials should also ideally be MRI-compatible because they are used during induction of anesthesia. They can be kept in an MRI-safe area outside the MRI scanner for easy access.

\section{Management of emergencies}

Any emergency that occurs in the MRI environment is more difficult to manage while the child is in the MRI scanner. Hence, it is recommended that if a medical emergency occurs in the scanner, the child should be immediately removed from zone IV while cardiopulmonary resuscitation is initiated, if indicated. The child should be transported to a location that has resuscitation equipment capabilities such as a defibrillator, code cart, resuscitation drugs, airway equipment, oxygen and suction. Airway emergencies such as aspiration, obstruction and laryngospasm are the most common emergencies that occur during an MRI scan. These can often be managed by an experienced pediatric anesthesiologist in the MRI suite if an anesthesia machine, medications and suction equipment are available inside the MRI suite. If the emergency becomes more severe, such as leading to cardiopulmonary arrest, the child should immediately be removed from the MRI scanner and appropriate resuscitation should be initiated. 


\section{Post-anesthesia care}

The anesthesiologist should collaborate with the radiology team to ensure safe and appropriate post-general anesthesia recovery of the pediatric patient. Pediatric patients receiving anesthesia within the MRI suite should have access to postanesthetic care that is consistent with that provided in other areas of the institution after general anesthesia, including a dedicated intensive care unit in situations of anesthetic emergencies such as airway events (i.e. aspiration). Recovery areas should enable close monitoring of vital signs and have oxygen, suction and resuscitation equipment available, as well as access to trained personnel. Children and parents should be provided with standard post-general anesthesia instructions for discharge.

\section{Strategies to mitigate sedation and anesthesia risks}

Several strategies that include the implementation of supportive programs such as child life and therapy dogs, joint staff efforts, use of tools that facilitate patient collaboration, ambient optimization and MRI-specific techniques can help mitigate the use of sedation and general anesthesia and their associated risks. When a child fails a non-sedated MRI despite these strategies, efforts can be made to repeat the examination under sedation/general anesthesia during the same day, or the study can be rescheduled to be repeated on a dedicated sedation MRI timeslot at a later date.
Child life specialists, therapy-dog programs and joint staff efforts

Child life specialists help prepare, coach, distract and support children in an attempt to increase the likelihood that a child can cooperate during the acquisition of MRI studies without sedation. These specialists have added significant value at many pediatric institutions [38]. The child life specialist might enter the scanner room (zone IV) to help the child get comfortable prior to the initiation of image-acquisition and can remain available in the MRI console (zone III) to re-enter the room in case the child needs further support. In many instances, the use of therapy dogs has helped minimize MRI anxiety in children [12] (Fig. 1). Hand-in-hand collaboration and constant communication among radiologists, anesthesiologists, technologists, child life specialists, nurses, schedulers and families is crucial for a successful performance of nonsedated MRI in young children [39, 40].

\section{Facilitating tools to increase patient collaboration}

Relaxation techniques, behavioral reinforcement and cognitive behavioral therapy can be useful to decrease anxiety, particularly among older children $[1,13,16]$. The use of MRIcompatible goggles to watch movies and MRI-compatible headphones to listen to music is also helpful $[1,12]$. Prescanning familiarity with the MR system through the use of photographs, educational videos and even a small-scale MRI model (e.g., LEGO bricks) can facilitate patients' collaboration [1, 12] (Fig. 2). The use of sound-attenuating materials such as earplugs and inner bore insulation of the magnet can improve patients' cooperation [1]. The use of MRI-
Fig. 1 Dog-therapy program. Clinical image of a child life specialist demonstrating how a dog lies on the MRI table (image used with staff permission)

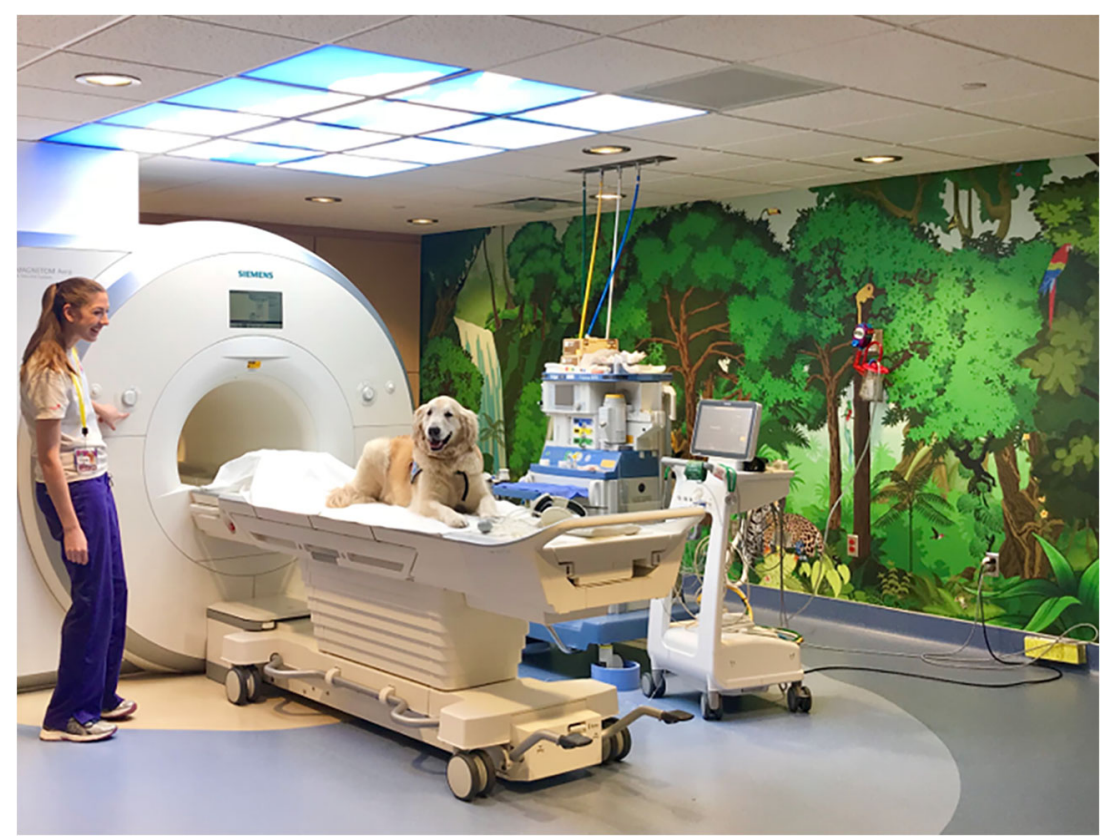




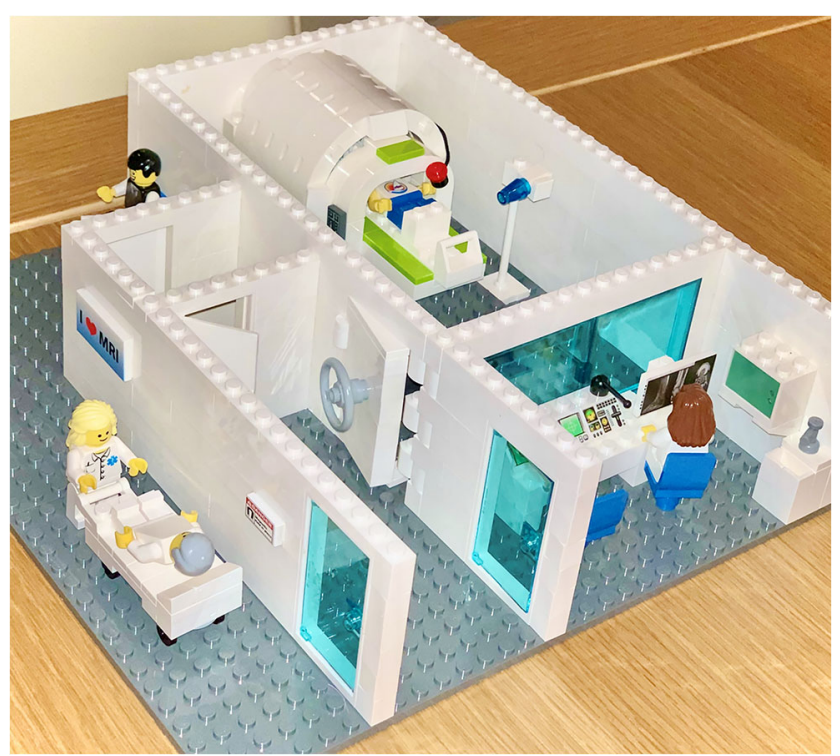

Fig. 2 MRI area prototype with LEGO bricks (I love MRI build your own MRI with LEGO bricks, an initiative lead by Dr. Ben Taragin from the Children's Hospital at Montefiore (USA), Dr. Erik Ranschaert from the Jeroen Bosch Hospital (The Neatherlands), and the Society for Pediatric Radiology

customized play therapy with pediatric patients undergoing diagnostic MRI has also been shown to significantly reduce the use of sedation [41] (Fig. 3). The use of immobilization devices is also critical, especially among infants $[1,14]$.

Another alternative is the use of a mock scanner or tunnel to prepare children for MRI examinations [1, 16] (Fig. 4). The mock scanner is a full-scale replica of an MRI system, without magnets, typically located within the department of radiology and in close proximity to the MRI suite [16]. To minimize the use of electrical and electronic fittings, the mock scanner is equipped with a manually operated patient table, plastic replica of a head coil, foam cushions, headphones, and earplugs.
Speakers inside the bore reproduce the sounds of various scan sequences that can be heard during actual MRI examinations.

\section{Local environment optimization}

Optimization of the local environment before and during MRI acquisition can be achieved with a feed-and-swaddle technique along with low-intensity lighting and minimal noise for neonates and young infants up to the age of 6 months [4, 42]. The feed-and-swaddle technique refers to the use of feeding and swaddling to induce natural sleep in infants because they tend to sleep when warmed and recently fed [14, 43]. Encouraging sleep deprivation and using melatonin hormones before imaging are other techniques that might improve the success of MRI studies with or without sedation [20,44]. The use of pacifiers and sucrose can be successful in neonates and infants younger than 3 months $[1,4]$. The success rate of sleep induced by feeding term children younger than 3 months may be up to $75 \%$ [45]. Decorated walls (murals), distraction methods, educational techniques, and mock imaging effectively improve rapport and decrease anxiety in older patients $[4,16,42]$ (Figs. 5 and 6).

\section{Techniques specific to magnetic resonance imaging}

Numerous MRI techniques that are fast and motioninsensitive are being incorporated in pediatric MRI protocols. The principles underlying all of these techniques are beyond the scope of this article; however, the reader is directed to recent review articles that summarize these methods [3, 46, 47]. These include techniques for undersampling $\mathrm{k}$-space to accelerate image acquisition, with or without advanced reconstruction algorithms to improve image quality of sparsely acquired datasets. In addition, prospective and retrospective
Fig. 3 Child life program and play therapy. A child life specialist uses a doll to show a child what will happen to the child's intravenous catheter during the MRI scan (precoronavirus disease [COVID] image used with permission from the child's family and staff)

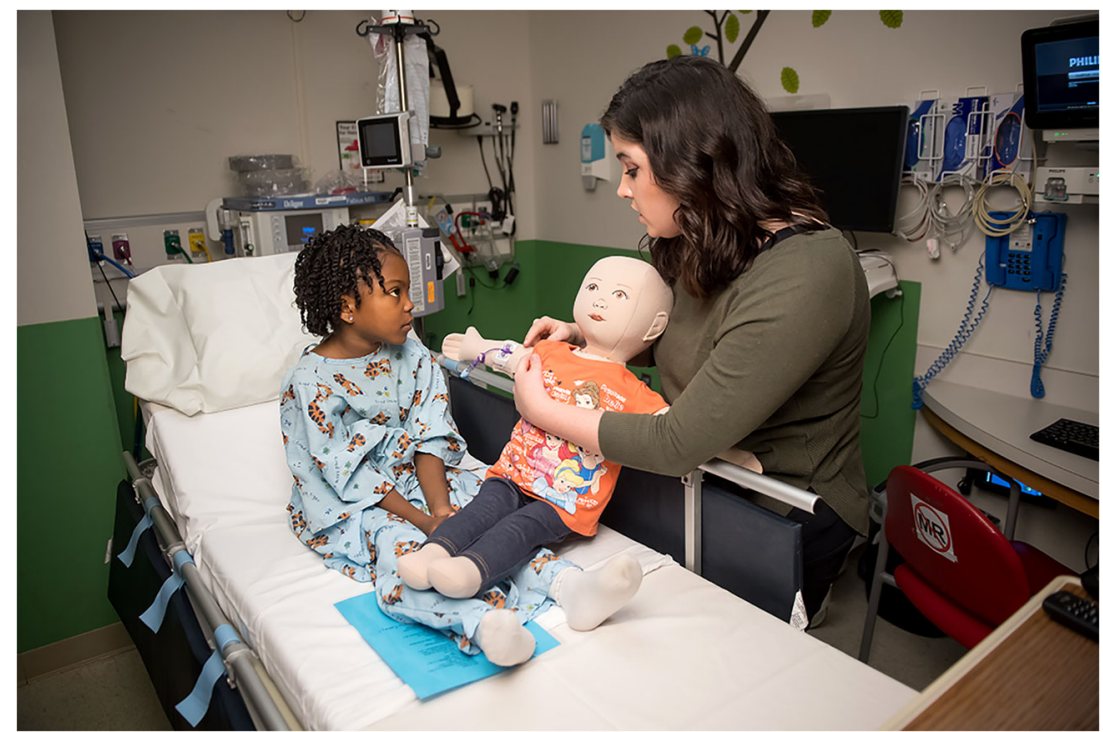


Fig. 4 Mock MRI. Clinical image shows the mock MRI scanner, which has the same size bore as the majority of 1.5-T and 3-T scanners. A child life specialist demonstrates coil placement using a doll. After the process is completed, depending on preference, the child can choose to go through the mock experience and enter the scanner bore (image used with staff permission)

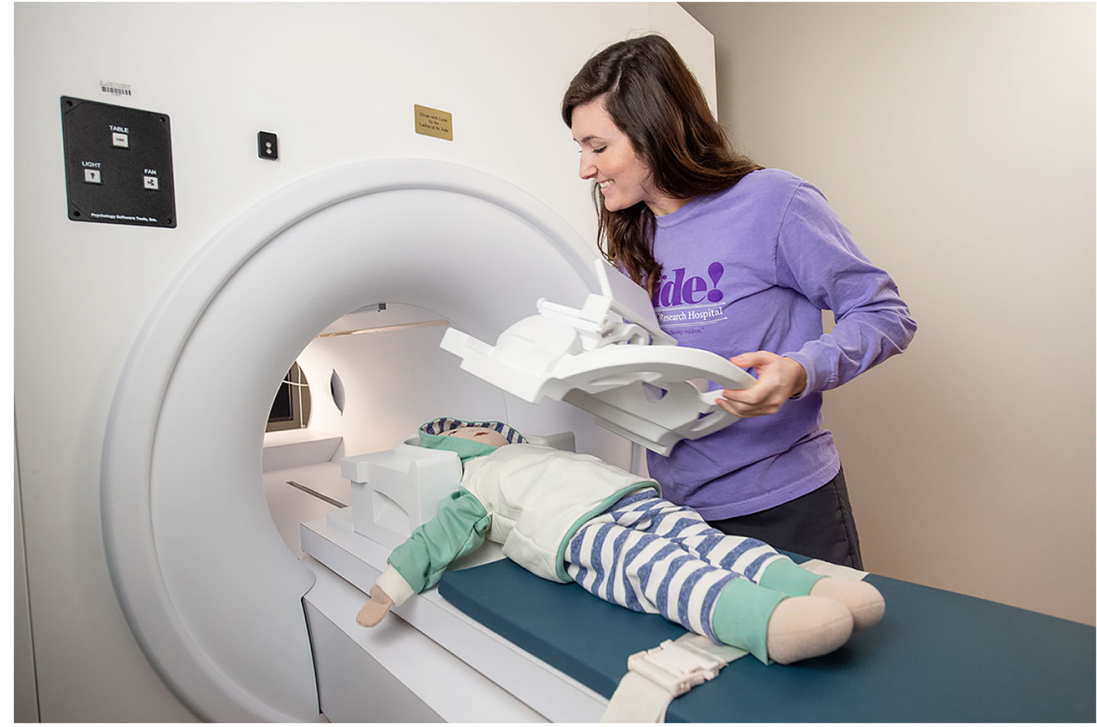

methods have been developed to compensate for or to correct motion degradation.

Parallel imaging methods enable undersampling of k-space through the use of phased-array coils with multiple receiver elements to assist spatial localization [48]. Parallel imaging acceleration is routinely used clinically to accelerate MR acquisition by not sampling some lines of $\mathrm{k}$-space in the phaseencoding direction while estimating missing information from coil sensitivity profiles of adjacent receiver elements. Parallel imaging usually leads to 2- to 3 -fold acceleration, although

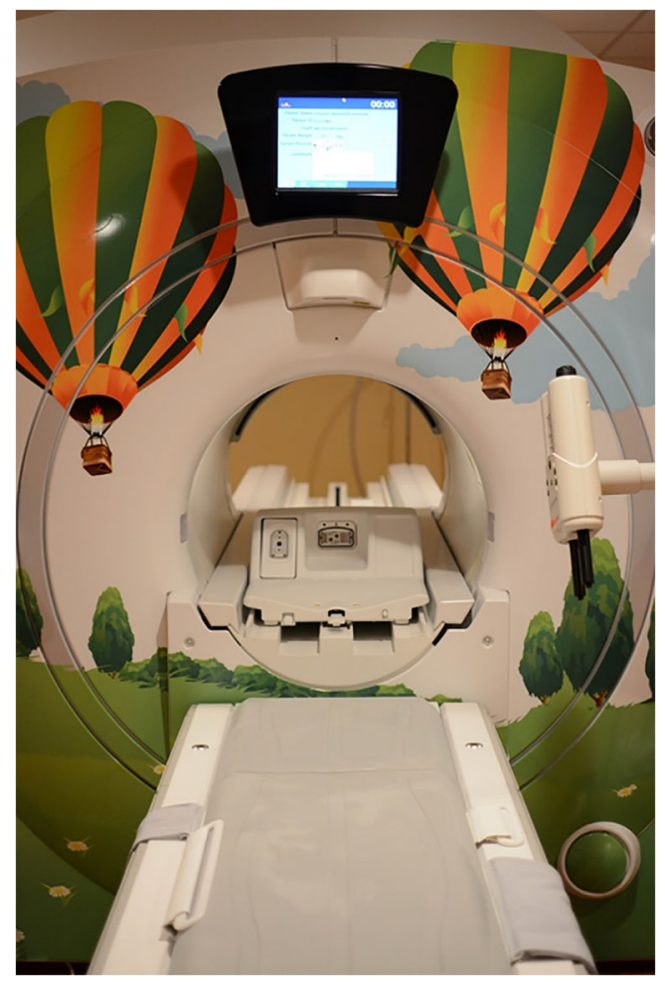

Fig. 5 Clinical image of MRI scanner decorations recently developed high channel count and flexible coils offer the promise of higher acceleration factors and are undergoing clinical validation for applications in the pediatric population $[49,50]$. Higher signal acquired with a 3 -tesla $(T)$ versus a 1.5 $\mathrm{T}$ scanner, combined with parallel imaging, has the potential to reduce the overall scan time and thus the need for sedation/ general anesthesia. The trade-offs in signal-to-noise ratio (SNR) with a higher parallel imaging factor can be offset by improved surface coil design and protocol optimization to reflect the higher signal from a 3-T scanner. Simultaneous multi-slice imaging utilizes a multiband radiofrequency transmission pulse that allows excitation and acquisition of imaging data from multiple slices simultaneously to accelerate image acquisition [51]. Simultaneous multi-slice technique has been shown to accelerate diffusion-weighted sequences by up to 2-fold in clinical studies [52]. Radial imaging techniques utilize radially oriented blades of $\mathrm{k}$-space sampling, in contrast to conventional Cartesian sequences that sample kspace in a row-by-row configuration [3]. This technique helps to reduce motion degradation artifacts because the phaseencoding direction is not coherent and motion artifact is dispersed along the rotating axis of the blades [53]. In addition, the radial configuration leads to oversampling of the center of k-space, which improves image contrast and SNR compared to Cartesian sampling (Fig. 7). Compressed sensing reconstruction is a technique that significantly decreases MR image acquisition time through k-space undersampling in an incoherent manner in combination with an iterative reconstruction algorithm to remove incoherent noise artifacts [54, 55]. Compressed sensing is being combined with radial kspace sampling to enable high temporal resolution multiphase dynamic contrast-enhanced imaging in pediatric patients who are free-breathing [56, 57]. These sequences are helpful for young children undergoing MRI awake who cannot suspend 
Fig. 6 Clinical image of MRI area wall decorations

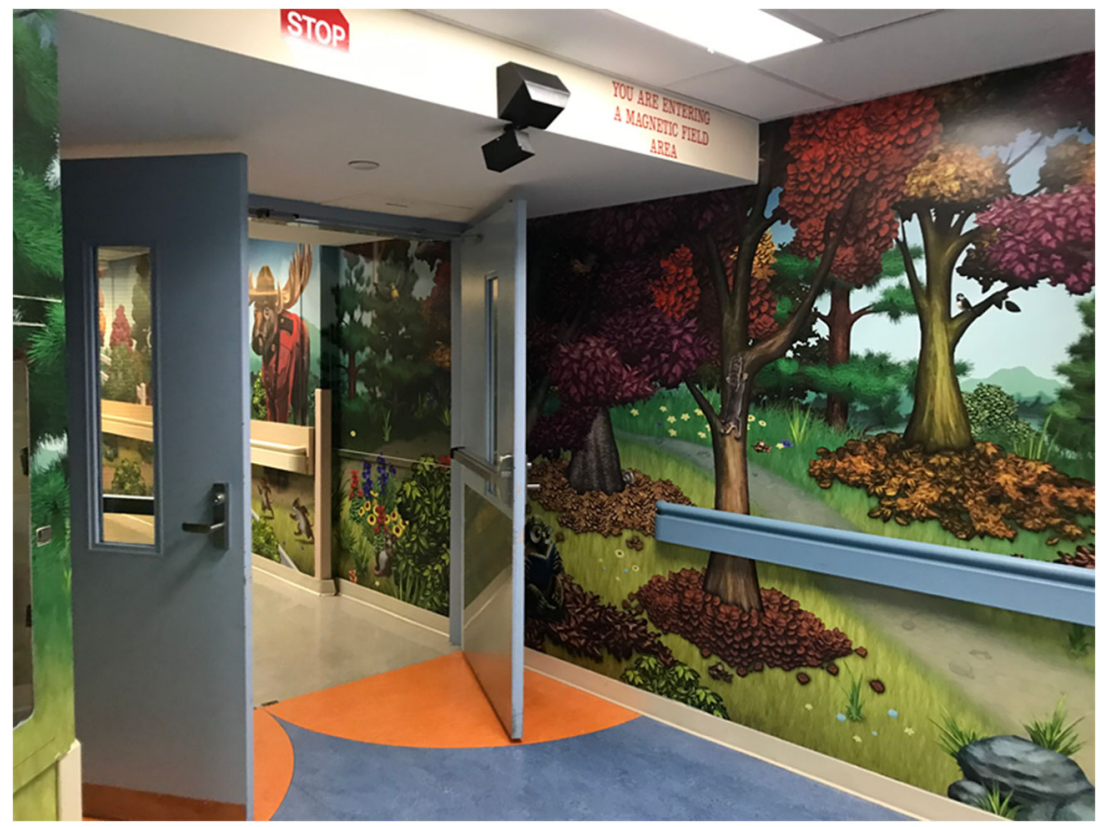

respiration, as well as children undergoing MRI with sedation who are allowed to breathe spontaneously. Finally, artificial intelligence and deep learning algorithms are also being developed that can restore diagnostic image quality associated with sparse or motion-degraded MR images [58].

\section{Tailored magnetic resonance protocols with appropriate coil selection}

While many adult radiology departments have developed a standardized set of adult MRI protocols, pediatric radiology departments need to generate diagnostic-quality MR images from patients spanning a wide range of sizes and phases of development. Challenges to pediatric MR protocol standardization can stem from multiple imaging sites, MR equipment vendors and field strengths, in addition to variations in experience among technologists, radiologists and anesthesiologists involved with pediatric MRI. These challenges are

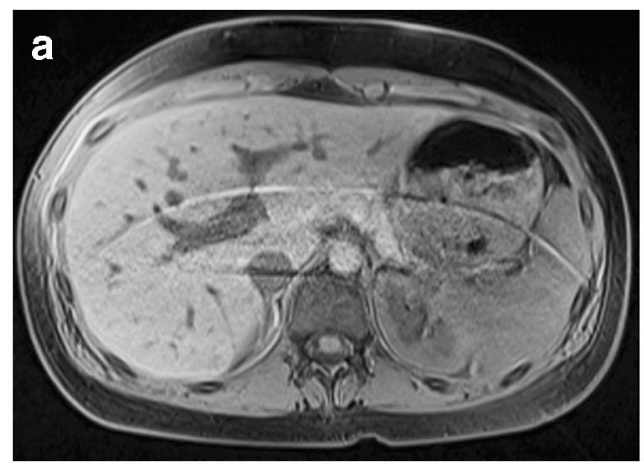

Fig. 7 Radially acquired imaging technique of the abdomen in a 16-yearold girl. a Axial T1-weighted gradient echo (GRE) MR image with fat saturation and breath-hold and conventional k-space sampling (scan time $=\sim 20 \mathrm{~s}$ ). $\mathbf{b}$ Axial T1-weighted GRE MR image with fat saturation and exacerbated by the need to have variations of MR protocols tailored to different patient sizes, the ability to suspend respiration (free breathing versus breath-hold acquisitions) as well as sedation needs (awake with child life specialist support versus sedation/general anesthesia). A structured approach to creating and standardizing pediatric MR protocols is crucial to ensure that the clinical question is answered in each case while minimizing unnecessary imaging and the use of sedation/ general anesthesia or contrast agents.

A recent study has shown a direct association between MR scan time and patient anesthetic medication exposure in children undergoing MRI with sedation [59], highlighting the importance of short MRI protocols that better conform to the definition of a short exposure to sedation and general anesthesia, as stated by the GAS study. Within a given protocol, the most important pulse sequences for lesion detection/ characterization and the most motion-sensitive sequences should be performed at the beginning of the scan. For children

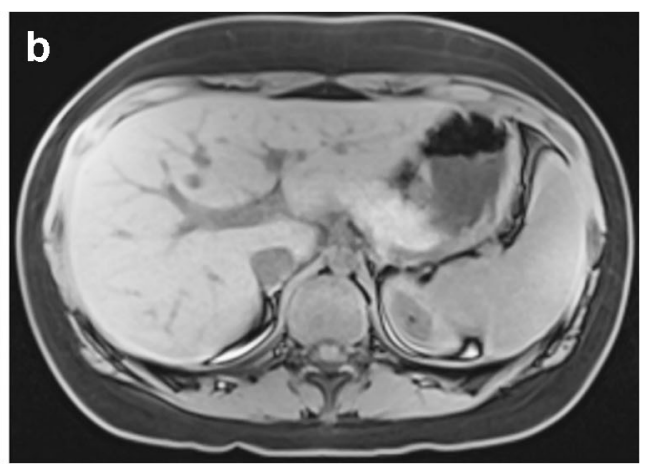

radial $\mathrm{k}$-space sampling (scan time $=\sim 3 \mathrm{~min}$ ). Breathing and parallel imaging artifacts are observed on the conventional image, whereas no such artifacts are identified on the radially acquired image 
undergoing non-sedated MRI, this increases the likelihood of a diagnostic study, while for the MRI under anesthesia, it minimizes the need for repeat images. The selection of an appropriate well-fitted coil, typically a phased-array coil, is also critical for optimizing the SNR in pediatric patients over a range of sizes [15] and specific to the body region of interest [1].

\section{Magnetic resonance imaging acoustic noise reduction}

Inherently, MRI scanners generate loud noises during the spatial encoding of the MR signal when current pulses are pushed through the gradient coil [60]. Because the gradient coil is placed inside a strong magnetic field, a pulsed Lorentz force that is induced causes vibrations of the coil structure and ultimately generates a compression wave in the air that is perceived as the "scanner noise" [60]. Depending on the acquisition method, current MRI systems report noise levels of 70$110 \mathrm{~dB}[61,62]$. The consequences of acoustic noise might manifest as increased stimulation in neonates, confusion in pediatric patients of other ages, and additional discomfort from increased hearing sensitivity caused by certain medications in sedated patients [63]. In addition, scanner manufacturers provide active noise-cancelling headphones. Studies have shown that the use of headphones during MRI examinations under general anesthesia reduces patients' spontaneous arm and leg movements significantly [64]. Newer methods such as construction of "quiet gradient coils," with applications of reduced gradient slew rate or compensation of net Lorentz force between current pathways, are now becoming clinically available [65]. These new acquisition methods also use a radial center-out sampling where endpoints of each spoke ultimately follow a spiral pattern. The gradient steps that are used in this sequence are relatively small in contrast to classic repetitive gradient ramp-up and -down steps during gradient-echo sequences, reducing gradient noise. The drawback to this method is a relative loss of image sharpness that might be perceived as increased noise or decreased SNR. As these new methods continue to evolve and become widely used, special patient groups, such as pediatric, claustrophobic and non-cooperative patients, are expected to benefit from MRI at ambient sound levels.

\section{Additional benefits of reducing the use of sedation and general anesthesia in pediatric magnetic resonance imaging}

In addition to reducing patient risks associated with sedatives and anesthetic drugs, the ability to perform MRI in children without these medications carries other benefits to patients, families, and the health care system at large. When performing MRI without sedation or anesthesia, the health care team's rapport with patients and parents/caregivers is improved [4] because the short-term effects (e.g., pain, anxiety, increased heart rate and blood pressure) and long-term effects (e.g., post-traumatic stress disorder, fear) that can be elicited during and after stressful medical procedures can be mitigated [13]. Visit and examination times, as well as financial costs associated with sedated/anesthetized MRI exams, are all reduced when pediatric MRI can be performed awake without sedatives/anesthetics [66]. Access to MRI is also improved because typically many more non-sedated MRI time slots are available relative to anesthesia appointments. Finally, safety and efficiency gains are associated with MRI performed without anesthesia. A recent study showed that the use of sedation/anesthesia for pediatric MRI was associated with higher rates of safety reports, including those related to service coordination issues such as patient transport, scan delays, and communication among care providers [67].

\section{Future directions to further decrease sedation and general anesthesia use in children}

The strategies we discussed, employed at different times before or during pediatric MRI scanning, greatly contribute to reducing patient anxiety and reliance upon sedation and general anesthesia. However, until high-quality diagnostic MRI can be acquired in infants, children and adolescents fast enough to avoid sedation or general anesthesia every time at every age, there is room to improve. Strategies in development or in the early stages of clinical adoption include advances in MR hardware and software technology described in the previous section. Another important area of innovation is in patient preparation and novel patient and family directed MRI training/preparation approaches. This includes MRI simulation using virtual reality (VR). This immersive experience will soon be leading the way in patient preparation through education and desensitization to the MRI environment, helping to alleviate anxiety related to claustrophobia, to reduce motion artifacts and potentially to avoid need for sedation and general anesthesia [68]. Ashmore et al. [69] demonstrated MRI VR to benefit parents and caregivers by improving their understanding of their child's MRI scan, thus reducing their own anxiety and facilitating patient cooperation. MRI VR simulates the child's MRI journey by replicating the time in the scanner using visuo-spatial and auditory input (Fig. 8). This often incorporates aspects of the MRI environment within a health care facility to further enhance familiarity.

Virtual reality in MRI is evolving along two paths. To date, the most common approach employs a basic MRI VR model. This typically relies on free downloadable applications such as ChildLife VR used on the patient or parent/caregiver's mobile device, in conjunction with a $360^{\circ}$ viewer, several of which 
are commercially available but can be as simple as a cardboard viewer [68-70]. This is similar to VR used in other complex medical settings, for example preparing a child for general anesthesia, adapted to be specific for MRI [70]. This is low cost, simple to use and widely available. Outcomes in a small cohort of children ages 4-12 years showed that general anesthesia during these children's MRI was avoided by $80 \%$ who would otherwise have required it [70]. Another distinct advantage of this basic MRI VR model over more complex systems is its ability to be used in the child's home, enabling the child to prepare for the MRI by using the VR multiple times for as long a period as required for the child to feel comfortable.

The more advanced approach to MRI VR builds on the basic model, merging it with biofeedback analysis. This uses sensors triangulated to the child's $360^{\circ}$ headset/viewer to instantaneously detect motion, which then triggers eventspecific instructions advising the patient to cease that action and lie still. Furthermore, biometric data such as pulse and respiratory rates can be gathered to assess stress response, in turn modulating information guiding the child. Physiologysensitive feedback combined with VR has been used in other environments to affect behavior change such as communication in children with autism and audiovisual feedback used to alter respiratory motion in cinematic MRI when measuring lung tumors [71, 72]. Biofeedback training in pediatric MRI VR is under investigation.

Comparison and validation of these differing VR approaches in pediatric MRI is a work in progress. Preliminary data and the use of MRI simulators - with complexity ranging from toys to full-scale scanners, yet all showing some benefit - suggest that both models are likely to be effective in improving patient compliance by reducing anxiety and motion, lessening the need for sedation and general anesthesia in children $[16,73,74]$. The optimum age range for MRI VR has

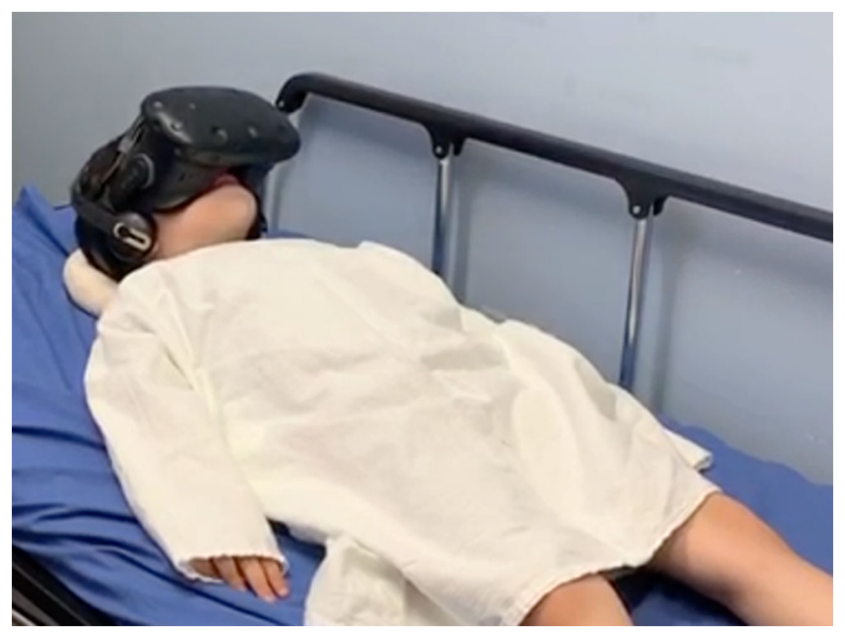

Fig. 8 Clinical photograph of a child using virtual reality (VR) goggles to simulate the MRI experience. Some VR systems are incorporating biofeedback capabilities not been established, although it is likely to be younger than 12 years [16, 69].

\section{Conclusion}

Sedation and general anesthesia have facilitated the widespread use of MRI examinations in the pediatric population. Knowledge of the sedation and general anesthesia safety profile, particularly in children younger than 3 years, is critical to proactively modify the approaches on a case-basis (individual) or group-basis (infants, young children and older children) by using complementary strategies that can ultimately improve patient care by enabling safer procedures, improved workflows and decreased overall costs.

Acknowledgments We would like to thank Stephanie Price, child life specialist at Children's Health Medical Center, for her dog-therapy program picture contribution, Shandra Taylor and Libby Gaitskill, child life specialists at St. Jude Children's Research Hospital, for their child play and mock MR pictures contribution, and pediatric radiologist Hillel S. Maresky, MD, from Temple University, for his MRI virtual reality model picture contribution. We would also like to thank Cherise Guess, PhD, ELS, senior editor at St. Jude Children's Research Hospital, for her help with editing this article.

\section{Declarations}

Conflicts of interest None

\section{References}

1. Dong S-Z, Zhu M, Bulas D (2019) Techniques for minimizing sedation in pediatric MRI. J Magn Reson Imaging 50:1047-1054

2. De Amorim e Silva CJT, Mackenzie A, Hallowell LM et al (2006) Practice MRI: reducing the need for sedation and general anaesthesia in children undergoing MRI. Australas Radiol 50:319-323

3. Kozak BM, Jaimes C, Kirsch J, Gee MS (2020) MRI techniques to decrease imaging times in children. Radiographics 40:485-502

4. Janos S, Schooler GR, Ngo JS, Davis JT (2019) Free-breathing unsedated MRI in children: justification and techniques. J Magn Reson Imaging 50:365-376

5. Cravero JP, Blike GT, Beach M et al (2006) Incidence and nature of adverse events during pediatric sedation/anesthesia for procedures outside the operating room: report from the pediatric sedation research consortium. Pediatrics 118:1087-1096

6. Davidson AJ, Morton NS, Arnup SJ et al (2015) Apnea after awake regional and general anesthesia in infants: the general anesthesia compared to spinal anesthesia study - comparing apnea and neurodevelopmental outcomes, a randomized controlled trial. Anesthesiology 123:38-54

7. Davidson AJ, Disma N, de Graaff JC et al (2016) Neurodevelopmental outcome at 2 years of age after general anaesthesia and awake-regional anaesthesia in infancy (GAS): an international multicentre, randomised controlled trial. Lancet 387:239-250

8. McCann ME, de Graaff JC, Dorris L et al (2019) Neurodevelopmental outcome at 5 years of age after general anaesthesia or awake-regional 
anaesthesia in infancy (GAS): an international, multicentre, randomised, controlled equivalence trial. Lancet 393:664-677

9. Flick RP, Katusic SK, Colligan RC et al (2011) Cognitive and behavioral outcomes after early exposure to anesthesia and surgery. Pediatrics 128:e1053-e1061

10. United States Food and Drug Administration (2019) FDA drug safety communication: FDA review results in new warnings about using genderal anesthetics and sedation durgs in young children and pregnant women. Online document. https:/www.fda.gov/drugs/ drug-safety-and-availability/fda-drug-safety-communication-fdareview-results-new-warnings-about-using-general-anesthetics-and. Accessed 9 Sept 2020

11. Clausen NG, Kähler S, Hansen TG (2018) Systematic review of the neurocognitive outcomes used in studies of paediatric anaesthesia neurotoxicity. Br J Anaesth 120:1255-1273

12. Perez M, Cuscaden C, Somers JF et al (2019) Easing anxiety in preparation for pediatric magnetic resonance imaging: a pilot study using animal-assisted therapy. Pediatr Radiol 49:1000-1009

13. Rothman S, Gonen A, Vodonos A et al (2016) Does preparation of children before MRI reduce the need for anesthesia? Prospective randomized control trial. Pediatr Radiol 46:1599-1605

14. Windram J, Grosse-Wortmann L, Shariat M et al (2012) Cardiovascular MRI without sedation or general anesthesia using a feed-and-sleep technique in neonates and infants. Pediatr Radiol 42:183-187

15. Jaimes C, Kirsch JE, Gee MS (2018) Fast, free-breathing and motion-minimized techniques for pediatric body magnetic resonance imaging. Pediatr Radiol 48:1197-1208

16. Carter AJ, Greer M-LC, Gray SE, Ware RS (2010) Mock MRI: reducing the need for anaesthesia in children. Pediatr Radiol 40: $1368-1374$

17. Harned RK 2nd, Strain JD (2001) MRI-compatible audio/visual system: impact on pediatric sedation. Pediatr Radiol 31:247-250

18. Arlachov Y, Ganatra RH (2012) Sedation/anaesthesia in paediatric radiology. Br J Radiol 85:e1018-e1031

19. Uffman JC, Tumin D, Raman V et al (2017) MRI utilization and the associated use of sedation and anesthesia in a pediatric ACO. J Am Coll Radiol 14:924-930

20. Pasini AM, Marjanović J, Roić G et al (2018) Correction to: melatonin as an alternative sedation method during magnetic resonance imaging in preschool children with musculoskeletal problems. Eur J Pediatr 177:1363-1366

21. Xu HS, Cavaliere RM, Min RJ (2020) Transforming the imaging experience while decreasing sedation rates. J Am Coll Radiol 17: 46-52

22. Brambrink AM, Evers AS, Avidan MS et al (2012) Ketamineinduced neuroapoptosis in the fetal and neonatal rhesus macaque brain. Anesthesiology 116:372-384

23. Creeley C, Dikranian K, Dissen G et al (2013) Propofol-induced apoptosis of neurones and oligodendrocytes in fetal and neonatal rhesus macaque brain. Br J Anaesth 110:i29-i38

24. Creeley CE, Dikranian KT, Dissen GA et al (2014) Isofluraneinduced apoptosis of neurons and oligodendrocytes in the fetal rhesus macaque brain. Anesthesiology 120:626-638

25. Davidson AJ, Sun LS (2018) Clinical evidence for any effect of anesthesia on the developing brain. Anesthesiology 128:840-853

26. Disma N, O'Leary JD, Loepke AW et al (2018) Anesthesia and the developing brain: a way forward for laboratory and clinical research. Paediatr Anaesth 28:758-763

27. Jevtovic-Todorovic V (2018) Exposure of developing brain to general anesthesia: what is the animal evidence? Anesthesiology 128: 832-839

28. Banerjee P, Rossi MG, Anghelescu DL et al (2019) Association between anesthesia exposure and neurocognitive and neuroimaging outcomes in long-term survivors of childhood acute lymphoblastic leukemia. JAMA Oncol. https://doi.org/10.1001/jamaoncol.2019. 1094
29. Ishida S, Kuratani N (2019) Association of anesthesia care and cognitive outcomes in survivors of childhood acute lymphoblastic leukemia. JAMA Oncol. https://doi.org/10.1001/jamaoncol.2019. 4897

30. Colquhoun DA, Mathis MR (2019) Association of anesthesia care and cognitive outcomes in survivors of childhood acute lymphoblastic leukemia. JAMA Oncol. https://doi.org/10.1001/jamaoncol. 2019.4900

31. Cravero JP, Beach ML, Blike GT et al (2009) The incidence and nature of adverse events during pediatric sedation/anesthesia with propofol for procedures outside the operating room: a report from the Pediatric Sedation Research Consortium. Anesth Analg 108: 795-804

32. Beach ML, Cohen DM, Gallagher SM, Cravero JP (2016) Major adverse events and relationship to nil per os status in pediatric sedation/anesthesia outside the operating room: a report of the Pediatric Sedation Research Consortium. Anesthesiology 124:80 88

33. Society for Pediatric Sedation Research (2019) Research: Pediatric Sedation Research Consortium. Webpage. https://www. pedsedation.org/resources/research/. Accessed 17 Jun 2020

34. Kurth CD, Coté CJ (2015) Postoperative apnea in former preterm infants general anesthesia or spinal anesthesia - do we have an answer? Anesthesiology 123:15-17

35. Emrath ET, Stockwell JA, McCracken CE et al (2014) Provision of deep procedural sedation by a pediatric sedation team at a freestanding imaging center. Pediatr Radiol 44:1020-1025

36. Jenkins E, Hebbar KB, Karaga KK et al (2017) Experience with the use of propofol for radiologic imaging in infants younger than 6 months of age. Pediatr Radiol 47:974-983

37. Cravero JP (2012) Pediatric sedation with propofol - continuing evolution of procedural sedation practice. J Pediatr 160:714-716

38. Koch BL (2008) Avoiding sedation in pediatric radiology. Pediatr Radiol 38:S225-S226

39. Robertson RL, Silk S, Ecklund K et al (2018) Imaging optimization in children. J Am Coll Radiol 15:440-443

40. Jaimes C, Robson CD, Machado-Rivas F et al (2020) Success of non-sedated neuroradiological MRI in children 1 to 7 years old. AJR Am J Roentgenol. https://doi.org/10.2214/AJR.20.23654

41. Bharti B, Malhi P, Khandelwal N (2016) MRI customized play therapy in children reduces the need for sedation - a randomized controlled trial. Indian J Pediatr 83:209-213

42. Greer M-LC, Vasanawala SS (2020) Invited commentary: reducing sedation and anesthesia in pediatric patients at MRI. Radiographics 40:503-504

43. Antonov NK, Ruzal-Shapiro CB, Morel KD et al (2017) Feed and wrap MRI technique in infants. Clin Pediatr 56:1095-1103

44. Sanborn PA, Michna E, Zurakowski D et al (2005) Adverse cardiovascular and respiratory events during sedation of pediatric patients for imaging examinations. Radiology 237:288-294

45. Nicolson SC, Schreiner MS (1994) Feed the babies. Anesth Analg 79:407-409

46. Serai SD, Hu HH, Ahmad R et al (2020) Newly developed methods for reducing motion artifacts in pediatric abdominal MRI: tips and pearls. AJR Am J Roentgenol 214:1042-1053

47. Krishnamurthy R, Wang DJJ, Cervantes B et al (2019) Recent advances in pediatric brain, spine, and neuromuscular magnetic resonance imaging techniques. Pediatr Neurol 96:7-23

48. Glockner JF, Hu HH, Stanley DW et al (2005) Parallel MR imaging: a user's guide. Radiographics 25:1279-1297

49. Winkler SA, Corea J, Lechêne B et al (2019) Evaluation of a flexible 12-channel screen-printed pediatric MRI coil. Radiology 291: $180-185$

50. Zhang T, Grafendorfer T, Cheng JY et al (2016) A semiflexible 64channel receive-only phased array for pediatric body MRI at $3 \mathrm{~T}$. Magn Reson Med 76:1015-1021 
51. Feinberg DA, Setsompop K (2013) Ultra-fast MRI of the human brain with simultaneous multi-slice imaging. J Magn Reson 229: 90-100

52. Obele CC, Glielmi C, Ream J et al (2015) Simultaneous multislice accelerated free-breathing diffusion-weighted imaging of the liver at 3T. Abdom Imaging 40:2323-2330

53. Pipe JG (1999) Periodically rotated overlapping parallel lines with enhanced reconstruction (PROPELLER) MRI: application to motion correction. In: Proceedings of the International Society of Magnetic Resonance in Medicine, pp 242-743

54. Vasanawala SS, Alley MT, Hargreaves BA et al (2010) Improved pediatric MR imaging with compressed sensing. Radiology 256: 607-616

55. Lustig M, Donoho D, Pauly JM (2007) Sparse MRI: the application of compressed sensing for rapid MR imaging. Magn Reson Med $58: 1182-1195$

56. Chandarana H, Feng L, Ream J, Wang A (2015) Respiratory motion-resolved compressed sensing reconstruction of freebreathing radial acquisition for dynamic liver MRI. Investig Radiol 50:749-756

57. Cheng JY, Zhang T, Ruangwattanapaisarn N et al (2015) Freebreathing pediatric MRI with nonrigid motion correction and acceleration. J Magn Reson Imaging 42:407-420

58. Zhu B, Liu JZ, Cauley SF et al (2018) Image reconstruction by domain-transform manifold learning. Nature 555:487-492

59. Machado-Rivas F, Leitman E, Jaimes C et al (2020) Predictors of anesthetic exposure in pediatric MRI. AJR Am J Roentgenol. https://doi.org/10.2214/AJR.20.23601

60. Mansfield P, Glover PM, Beaumont J (1998) Sound generation in gradient coil structures for MRI. Magn Reson Med 39:539-550

61. McJury M, Blug A, Joerger C et al (1994) Acoustic noise levels during magnetic resonance imaging scanning at $1.5 \mathrm{~T}$. Br J Radiol $67: 413-415$

62. McJury MJ (1995) Acoustic noise levels generated during high field MR imaging. Clin Radiol 50:331-334

63. Quirk ME, Letendre AJ, Ciottone RA, Lingley JF (1989) Anxiety in patients undergoing MR imaging. Radiology 17:463-466
64. Oğurlu M, Orhan ME, Çinar S et al (2012) Effect of headphones on sevoflurane requirement for MRI. Pediatr Radiol 42:1432-1436

65. Alibek S, Vogel M, Sun W et al (2014) Acoustic noise reduction in MRI using silent scan: an initial experience. Diagn Interv Radiol 20:360-363

66. Vanderby SA, Babyn PS, Carter MW et al (2010) Effect of anesthesia and sedation on pediatric MR imaging patient flow. Radiology 256:229-237

67. Jaimes C, Murcia DJ, Miguel K et al (2018) Identification of quality improvement areas in pediatric MRI from analysis of patient safety reports. Pediatr Radiol 48:66-73

68. Brown RKJ, Petty S, O'Malley S et al (2018) Virtual reality tool simulates MRI experience. Tomography 4:95-98

69. Ashmore J, Di Pietro J, Williams K et al (2019) A free virtual reality experience to prepare pediatric patients for magnetic resonance imaging: cross-sectional questionnaire study. JMIR Pediatr Parent 2: e11684

70. O'Sullivan B, Alam F, Matava C (2018) Creating low-cost 360degree virtual reality videos for hospitals: a technical paper on the dos and don'ts. J Med Internet Res 20:e239

71. Kuriakose S, Lahiri U (2017) Design of a physiology-sensitive VRbased social communication platform for children with autism. IEEE Trans Neural Syst Rehabil Eng 25:1180-1191

72. Lee D, Greer PB, Ludbrook J et al (2016) Audiovisual biofeedback improves cine-magnetic resonance imaging measured lung tumor motion consistency. Int J Radiat Oncol Biol Phys 94:628-636

73. Pressdee D, May L, Eastman E, Grier D (1997) The use of play therapy in the preparation of children undergoing MR imaging. Clin Radiol 52:945-947

74. Hallowell LM, Stewart SE, de Amorim E Silva CT, Ditchfield MR (2008) Reviewing the process of preparing children for MRI. Pediatr Radiol 38:271-279

Publisher's note Springer Nature remains neutral with regard to jurisdictional claims in published maps and institutional affiliations. 